\title{
基于海洋能的海水资源综合利用研究
}

\author{
李伟，杨易嘉，顾亚京，刘宏伟，林勇刚，张南强 \\ (浙江大学流体动力与机电系统国家重点实验室，杭州 310027)
}

\begin{abstract}
摘要: 本文将海洋能发电技术、海水淡化技术与浓盐海水电解制氢技术相结合, 提出了一种综合利用海洋能源和海水资源的 新模式。海洋能发电设备能为海岛和海上设施提供电能, 在通过海水制淡有效解决海岛或海上设施供水问题的同时也会产生 副产品浓盐水一一目前均作为废弃物回放大海, 不仅成为一种污染源, 也造成海水浓盐水中富含的氢、氯、钠等化工资源的 浪费。因此本文进一步研究海洋能电解浓盐水制氢、制碱技术, 在实现海能海用、海水资源化利用的同时, 也为建设海洋补 氢站提供了可能。
\end{abstract}

关键词: 潮流能; 海洋化学能; 海水综合利用; 制氢; 制碱

中图分类号: P746.1 文献标识码：A

\section{Comprehensive Utilization of Seawater Resources Based on Ocean Energy}

\author{
Li Wei, Yang Yijia, Gu Yajing, Liu Hongwei, Lin Yonggang, Zhang Nanqiang
}

(State Key Laboratory of Fluid Power \& Mechatronic Systems, Zhejiang University, Hangzhou 310027, China)

\begin{abstract}
This study combines the tidal current power generation system with seawater desalination and hydrogen production by concentrated seawater electrolysis, and proposes a comprehensive utilization mode of marine energy and seawater resources. Tidal turbines can provide sufficient power for islands or offshore facilities, while seawater desalination can supply adequate freshwater to islands. However, concentrated seawater, a byproduct of seawater desalination, is usually treated as waste and discharged back to the ocean. It thus becomes a pollution source and meanwhile causes a waste of chemical resources enriched in it, such as hydrogen, chlorine, and sodium. Therefore, this paper further studies the technologies for producing hydrogen and caustic soda by concentrated seawater electrolysis using the tidal current power generation system. By this means, marine energy resources are exploited for marine use, the seawater resource is properly recycled, and the establishment of offshore hydrogen supply stations may become possible.

Keywords: tidal current energy; marine chemical energy; comprehensive utilization of seawater; hydrogen production; caustic soda production
\end{abstract}

\section{一、研究背景}

海洋能根据存在形式的不同, 可以分为潮汐能、 潮 (洋) 流能、波浪能、盐差能、温差能等, 这些
狭义海洋能的理论储量高达 $2.1 \times 10^{6} \mathrm{TWh} / \mathrm{a}[1]$, 大 大超出了目前人类利用各种能量 (功率) 的总和。 此外还有广义的海洋能, 如海上风能、海洋生物质 能、海洋地热能等。上述狭义或广义海洋能均为可

收稿日期 : 2019-10-12; 修回日期 : 2019-10-19

通讯作者: 李伟, 浙江大学流体动力与机电系统国家重点实验室教授, 研究方向为可再生能源装备与应用系统（风力、海流、波浪发电）、 机电控制与测试技术等; E-mail: 1iw@zju.edu.cn

资助项目：中国工程院咨询项目 “海洋强国战略研究 2035” (2018-ZD-08)

本刊网址：www.engineering.org.cn/ch/journal/sscae 
再生的清洁能源。然而, 在海水中还蕴含了数量巨 大的 “海洋 (水) 化学能”。有效利用这种特殊的 能量并将海量的海水 “资源化”, 是本文拟提出的 研究新领域、技术新问题。具体而言, 本文将着重 讨论一个有研究价值的海洋能、海水淡化、海水资 源综合利用新方案, 即通过海洋能发电进行海水淡 化, 在获得宝贵淡水资源的同时, 进一步利用海洋 能转化的电力完成制淡副产品浓盐水的电解制氢、 制碱工艺, 从海水中直接获取氢气、氯气、纯碱、 烧碱等化学制品, 从而完成向大海要能源、向大海 要淡水、向大海要资源的全过程, 开拓海洋能与海 水资源综合利用的新途径。

海水制淡是解决沿海（近海）地区淡水资源短 缺的现实选择, 具有重要的社会意义和经济价值, 海水淡化技术本身产业已成熟。利用海洋能实现海 能海用、就地取能、就近发电, 实现沿海缺水地区 或无水岛屿、岛礁的海水淡化供给, 进一步实现了 海上可再生能源技术与海水淡化技术的融合，拓展 了产业链, 可望进一步降低海水淡化的成本。海水 淡化后的浓盐水中的氯化钠浓度与电解盐工艺溶液 中氯化钠的浓度已经非常接近。在海洋能发电技术 方面, 包括浙江大学、中国科学院广州能源研究所 等单位在内的国内外研究机构和企业在海洋能发电 领域做了长期的、大量的工作，潮流能、波浪能等 发电装备与技术日趋成熟。但是同时利用海洋能产 生的电力和海水制淡产生的副产品浓盐水, 来完成 通常在内陆厂家消耗大量电力的电化工生产过程是 一个新概念, 目前尚无直接、具体的研究和实施案 例。因此下文将分析上述方案中的利用海洋能发电 和海水制淡剩余浓盐水（通常回弃大海）两种海上 资源, 通过电解制氢及生产其他化工产品的工艺、 成本及应用技术等问题，探讨这一方案的可行性、 有效性，评估其潜在的经济、社会效益。

\section{二、海水淡化与其浓盐水副产品资源化利用 可行性分析}

中国是一个淡水资源缺乏的国家，在全国 660 多 个城市中，有 400 多个城市缺水，其中 108 个为严 重缺水城市 [2]。水资源缺乏已经成为制约社会经 济发展的重要因素。利用海水淡化技术获取淡水可
以不受时空和气候影响, 水质好、价格合理, 可以 为沿海地区提供稳定的市政供水与工业用水。然而 随着大规模反渗透膜法海水淡化工程的实施, 其副 产品浓海水的排放问题引起了广泛关注。浓海水不 仅含盐量高, 而且在海水预处理过程中会引入一 些化学物质, 如果处理不当会对环境造成影响甚 至污染 [3], 特别是对 (半) 封闭海域生态环境的 影响尤为突出。因此, 中国政府高度重视海水综合 利用，在《关于加快发展海水淡化产业的意见》（国 办发〔2012〕13 号）总体思路中，明确提出 “海 水淡化处理与资源综合利用相结合”。当 2020 年海 水淡化 $6 \times 10^{6} \sim 8 \times 10^{6} \mathrm{t} / \mathrm{d}$ 目标实现时，浓海水资 源化产业的产值将达到 370 亿元以上，不仅可获得 很好的综合经济效益, 而且将为解决国内急缺矿物 的来源, 以及保护海洋环境做出重要贡献, 其前景 非常广阔。以某 $1 \times 10^{5} \mathrm{t} / \mathrm{d}$ 海水淡化装置为例, 每 天排出的浓海水约为 $6 \times 10^{4} \mathrm{t}$, 其中含盐量约为 $6 \times 10^{3} \mathrm{t}$, 如果作为副产品提取就可以使海水淡化 成本降低 $20 \%$ [4]。该装置一年排出的浓海水约为 $2.19 \times 10^{7} \mathrm{t}$, 其中化学资源总量将超过 $2 \times 10^{6} \mathrm{t}$ 。 综上可见，直接将浓海水回放入海将造成资源的 浪费和海域的污染，而利用制淡浓海水则不仅是 海水资源综合利用链的延伸，也完全具备技术上 的可行性和创造新价值的潜力。

\section{三、基于潮流能或洋流能的海水资源综合利 用方案}

潮流能或洋流能是指海水在水平方向上流动所 具有的动能, 两者成因不尽相同。前者在海岸或者 岛屿附近最为集中, 对应流速大, 能量密度大。后 者在广阔大洋普遍存在, 通常流速偏小但总量极大。 潮流能或洋流能规律性强, 对海洋环境的影响较小, 很适合进行大规模开发 [5]。因此, 在海岸或者岛 屿上开展以潮流能发电为基础的海水资源综合利 用, 便于就地取能、海能海用, 具有极强的现实意 义和可能性。

\section{（一）基于潮流能的海水资源利用方式}

1. 基于潮流能发电的海水淡化

对于绝大部分远离大陆的海岛而言, 缺乏淡水 
资源是一个普遍的问题。常规的海水淡化方式离不 开电能的支持, 而岛屿的长距离输电往往有诸多限 制, 有时难以实现。而这些海岛附近往往具有天然 的潮流能或洋流能。因此, 通过潮（洋）流能发电 设备将其动能转化为电能, 再驱动海水淡化装置获 得淡水, 是解决远离大陆海岛淡水问题的有效方案。

2. 基于潮流能发电的海水浓盐水处理

前已叙及, 若将海水淡化所得的浓海水直接排 入大海中，不仅会对周围海域造成污染，而且还会 造成资源的极大浪费。海水中含有丰富的氯化钠, 是制备烧碱的重要原料。氢气、氯气作为制备烧碱 的副产物, 具有十分重要的经济价值, 尤其是氢气 作为氢燃料电池、氢能新能源汽车的能量来源, 其 重要价值不言而喻。

氯碱工业的核心部分为电解工序。据测算在 整个生产过程中, 电解工序所消耗的电能占到整体 能耗的 53.2\%, 许多制碱厂曾自备小型发电厂，在 满足生产过程中对电能消耗的同时又降低了生产成 本 [6]。然而自备电厂多为火力发电厂, 在发电过 程中对环境影响大, 电能消耗逐渐成为影响烧碱制 备的限制性因素。

这为将潮 (洋) 流能发电和氯碱工业相结合提 供了可能, 也为浓海水用于氯碱生产提供了便利。 将潮流能发电、海水淡化以及氯碱生产就地或就近 结合, 从而形成一套新的海洋能综合利用体系, 应 是海洋能开发利用历程上的重要一步。

\section{（二）基于潮（洋）流能的海水资源综合利用流程 分析}

图 1 为海水资源化综合利用流程图。原海水取 水经过一系列处理流程得到淡水、固碱产品、氢气、 氯气和浓盐酸等产物, 流程中电能均由潮（洋）流 能发电装备提供。

首先, 用反渗透膜法将预处理后的海水进行淡 化获得氯化钠浓度为 $6 \%$ \% $\%$ 的浓海水 [7], 反渗透 膜法具有投资低、能耗低等优点 [8]。

然后, 将得到的浓海水用电渗析法得到精致浓 海水。工业上通常采用电解饱和氯化钠溶液的方法 制备烧碱、氯气和氢气 [9], 故需要进行浓海水精 制提高溶液中氯化钠的含量。电渗析法作为成熟的 海水浓缩方法 [10], 可提高浓海水中氯化钠的浓度 以达到制碱的要求。

最后, 电解饱和氯化钠溶液制备烧碱、氯气和 氢气。在电解工序中, 精制浓海水从阳极侧进入离 子膜电解槽, 阴极侧加入部分海水淡化所得的淡水, 经电解后在阳极侧得到淡盐水和氯气, 在阴极侧得 到电解液和氢气。

阳极生成的淡盐水和氯气经过钛冷却器冷却后 再气液分离。流出的淡盐水经脱氯环节去除其中的 次氯酸, 可以在浓海水精制环节重复利用。氯气冷 却干燥后, 可以液化储存, 也可以与阴极生成的氢 气合成盐酸, 供电解工序使用。氢气干燥后可进入 高压储氢罐储存备用，也可进入盐酸生产工序。此

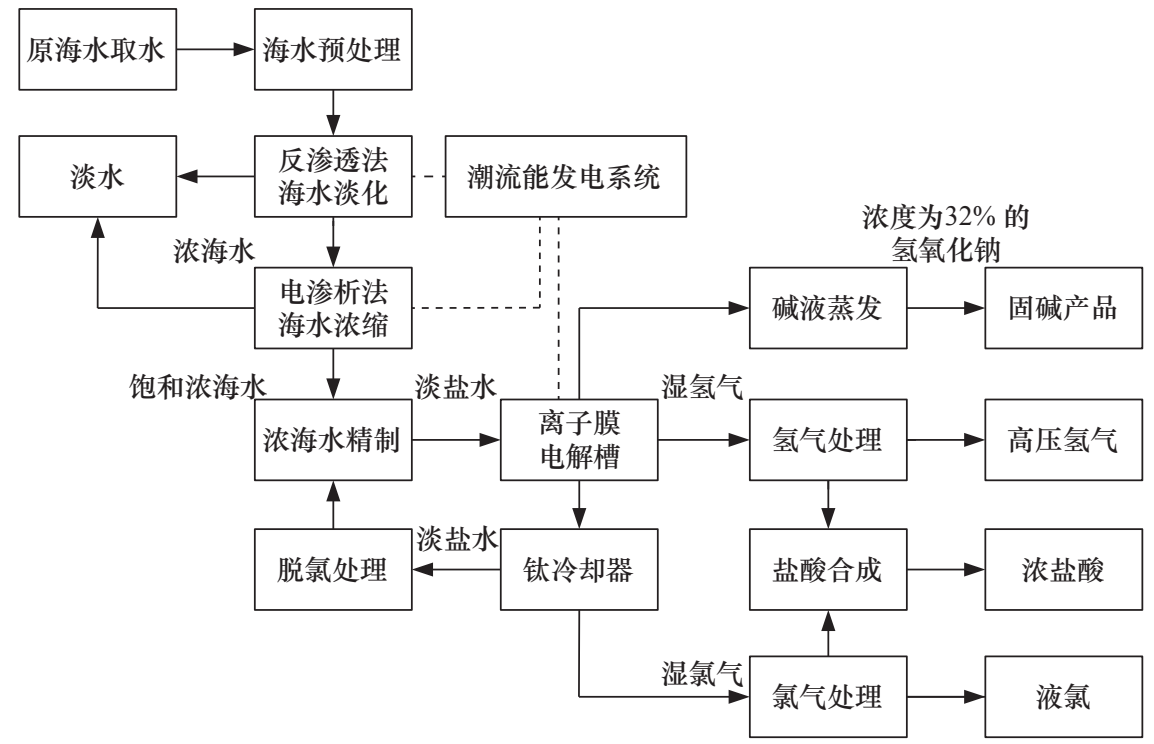

图 1 海水资源化综合利用流程图 
外, 将阴极所得的电解液蒸发, 便可获得固体烧碱 产品。

\section{（三）工艺运行经济型分析}

结合潮（洋）流能发电设备运行环境, 本文以 产能 $2.5 \times 10^{3} \mathrm{t} / \mathrm{d}$ 的海水淡化设备为例, $10 \mathrm{a}$ 为运行 时间期限，进行整套工艺流程的成本评估。本文所 提海水淡化工艺为目前成熟的反渗透膜法, 故其运 行成本与常规海水淡化设备无异。设备运行成本由 固定成本和经营成本两部分组成。海水淡化设备的 固定成本包括折旧、财务费用和其他费用; 经营成 本主要包括电费、维修费、膜更换费、药剂费、人 工费等 [11]。

海上风力发电系统与潮流能发电系统在投资运 维上有诸多相似之处, 故本文以海上风电的上网电 价来预估潮流能上网电价来进行成本计算具有较强 的参考性。由文献 [12] 可知, 根据《国家发展和改 革委员会关于调整光伏发电陆上风电标杆上网电价 的通知》（发改价格（2016２２２２号）近海风电项 目标杆上网电价为 0.85 元 $/ \mathrm{kW} \cdot \mathrm{h}$ 。结合文献 [11] 中的运行成本计算可知, 在本文设计的条件下, 海 水淡化的成本为 12.4 元 $/ \mathrm{t}$ 。

采用电渗析法来浓缩经海水淡化后的浓海水, 折合成氯化钠计算其用电量为 $170 \mathrm{~kW} \cdot \mathrm{h} / \mathrm{t}$ [13], 以 0.85 元 $/ \mathrm{kW} \cdot \mathrm{h}$ 电价来计算, 则获得氯化钠的用 电成本为 144.5 元 $/ \mathrm{t}$ 。而目前氯碱工业生产的原盐 价格在 250 元 $/ \mathrm{t}$ 左右, 故利用浓缩后的浓海水作为 氯碱生产的原液从一定程度上可降低原料成本。

产能为 $2.5 \times 10^{3} \mathrm{t} / \mathrm{d}$ 的海水淡化设备每天能产 生 $3 \times 10^{3} \mathrm{t}$ 浓海水, 故需要充分利用浓海水, 烧碱 产能需为 $5 \times 10^{4} \mathrm{t} / \mathrm{a}$ 。根据文献 [14], 离子膜电解 法制烧碱每吨电耗为 $2340 \mathrm{~kW} \cdot \mathrm{h}$ 、蒸汽消耗为 $0.9 \mathrm{t}$, 氯化钠消耗为 $1.6 \mathrm{t}$, 结合前文对电价和氯化钠价 格的折算, 得每吨烧碱的能耗成本为 2310 元。以 $10 \mathrm{a}$ 为时间期限, 加上设备折旧费及经营成本, 折 算每吨烧碱总成本约为 2594 元。

本文提出基于潮（洋）流能发电的海洋化学能 综合利用方案, 其海水淡化成本为 12.4 元 $/ \mathrm{t}$, 制烧 碱成本为 2594 元 $/ t$ 。而根据文献 [11] 和文献 [14] 中 海水淡化和制烧碱的成本计算, 可知常规条件下海 水淡化成本为 8.808 元 $/ t$, 制烧碱成本为 1281 元 $/ t$ 。
与常规的海水淡化、制烧碱工艺相比, 两者目前成 本差距的主要原因在于用电价格。而随着技术的进 步和规模的扩大，潮流能发电成本的降低和此方案 的经济价值将进一步显现。

\section{四、海水资源的下游应用}

饱和食盐水经电解能生成氢气、氯气、烧碱, 三者均是重要的化工原料。烧碱是氯碱工业的主要 产品, 具有重要的工业价值, 被誉为 “化工之母”。 氯气和氢气是氯碱工业重要的联产品, 对于氯碱工 业的发展起着关键作用。

烧碱广泛应用于洗涤剂、肥皇、造纸、印染、 纺织、医药、染料、金属制品、基础化工及有机化 工行业。国内烧碱需求结构基本保持稳定，下游消 费市场仍以氧化铝、造纸、印染、化纤等传统行业 为主 [15]。

氯气是氯碱工业重要的联产品, 应用十分广 泛, 是重要的自来水消毒剂, 也是漂白粉、漂白剂 的主要原料, 在电子工业大量应用于超大规模集成 电路制造。氯气下游产品也相当丰富。以氯气为源 头, 可氯醇化生产环氧丙烷, 下游生产聚醚和四氯 乙烯, 四氯乙烯可以部分生产制冷剂外售; 可生产 氯丙烯、环氧氯丙烷; 可生成光气, 再生产二苯 基甲烷二异氰酸酯 (MDI), 与另一条生产线的产 品配合可以进一步生产聚氨酯; 可以单独生产三氯 乙烯直接外售 [16]。

氢气在炼油方面和燃料电池方面应用广泛。其 对于提高轻油收率、改善油品品质具有重要的作用。 2017 年我国的炼油总量达 $7.7 \times 10^{8} \mathrm{t}$, 耗氢量约为 $6.16 \times 10^{6} \sim 1.078 \times 10^{7} \mathrm{t}[16]$ 。燃料电池具有巨大的 商业价值, 在交通、能源、军事和航天等领域前景 广阔。而氢燃料电池是燃料电池应用的重点方向。 以氢燃料电池为核心的氢燃料电池汽车、分布式发 电、应急电源等产业已经初现端倪 [17]。

此外, 氢气在深远海军民两用基地也发挥着巨 大的作用。据悉中国航天科技集团组建成立我国首 个军民融合氢能工程技术研发中心。若按本文提出 的海洋能制淡、制冰、制气和海水动能、化学能资 源化利用方案, 在包括我国近海和深远海依托海洋 能丰富的海岛岛礁完成发电、制淡、制氢装备系统, 
使其成为深远海自动化, 移动化, 无人化充电、充 水、充气的前沿供给基地和海水化工原材料、渔业 用冰生产储存基地, 不仅能推动可再生海洋能、氢 能利用等前沿领域高端技术装备的研究开发和工程 应用, 同时也能为维护我国海疆安全和海洋权益发 挥重大支撑保障作用。

\section{五、结语}

将潮流能发电和海水淡化、氯碱生产相结合, 因地制宜, 可充分利用海洋资源, 可减轻对陆地能 源的依赖, 并为在海岛和海上建立相关产业提供了 可能。

增加了潮流能的使用范围, 为潮流能使用提供 了重要的经济价值, 为潮流能发电产业化发展增添 了新的驱动力。

海水淡化获得的淡水可以为氯碱生产服务, 可 以为海岛提供生活用水, 也可以进行储存, 作为淡 水补给站为来往船只提供淡水。

将浓海水就地利用, 不但减少了海水淡化副产 物对环境的污染, 降低氯碱生产的成本, 又可充分 利用海洋化学能, 提高经济效益。

氢能是被公认的清洁能源, 被誉为 21 世纪最 具发展前景的二次能源。从海洋中获取氢气是未来 氢工业的发展方向之一, 通过本文中提出的海洋化 学能利用方案, 可制得高纯度氢气, 为建设海上补 氢站提供可能。

\section{参考文献}

[1] Ronger H H, Bart'hel F, Cabrera M, et al. Energy resources [M]. New York: United Nations Department of Economic and Social Affairs, World Energy Council, 2000.

[2] 中华人民共和国国家发展和改革委员会, 国家海洋局, 财政部. 海水利用专项规划 [R]. 北京: 中华人民共和国国家发展和改革 委员会, 国家海洋局, 财政部, 2005.

State Development and Reform Commission, State Oceanic Administration, Ministry of Finance of the PRC. Special plan for seawater utilization [R]. Beijing: State Development and Reform Commission, State Oceanic Administration, Ministry of Finance of the PRC, 2005.

[3] Ahmed H, Muneer H. Impact of desalination plants fluid effluents on the integrity of seawater, with the Arabian Gulf in perspective [J]. Desalination, 2005, 182: 373-393.

[4] 赵华, 刀远清. 利用核能海水淡化初探 [J]. 海洋科学, 2002, 26(7): $18-21$
Zhao H, DiaoY Q. Preliminary study on the use of nuclear energy seawater desalination [J]. Ocean Science, 2002, 26(7): 18-21.

[5] 孙志峰. 国内外海洋能利用技术发展现状 [C]. 海口: 深海能源 大会, 2015 .

Sun Z F. Development status of marine energy utilization technology at home and abroad [C]. Haikou: Deep Sea Energy Conference, 2015.

[6] 刘启照. 电解法烧碱生产中的能耗及节能技术 [J]. 氯碱工业, 2002 (8): 1-8.

Liu Q Z. Energy consumption and energy saving technology in electrolytic caustic soda production[J]. Chlor-Alkali Industry, 2002 (8): 1-8.

[7] 麻炳辉, 白永浩. 海水淡化后浓海水工厂化制盐浅析 [J]. 盐业 与化工, 2013, 42(4): 25-26.

Ma B H, Bai Y H. Analysis of salt production from concentrated seawater after seawater desalination [J]. Journal of Salt and Chemical Industry, 2013, 42(4): 25-26.

[8] 王宏涛, 李保安, 刘兵. 海水淡化技术现状及新技术评述 [J]. 盐 业与化工, 2014, 43(6): 1-4.

Wang H T, Li B A, Liu B. Review of the status quo and new technology of seawater desalination technology [J]. Journal of Salt and Chemical Industry, 2014, 43(6): 1-4.

[9] 常喜斌, 钟建云, 王冀锋. 离子膜制碱淡盐水的高效利用技术 [J]. 氯碱工业, 2017, 53(1): 8-11.

Chang X B, Zhong J Y, Wang J F. Efficient utilization technology of ion membrane alkali light brine [J]. Chlor-alkali Industry, 2017, 53(1): 8-11.

[10] 刘多阳, 宋剑. 电渗析法浓缩海水制盐的应用及发展 [J]. 盐科 学与化工, 2018 (3): 11-13.

Liu D Y, Song J. Application and development of concentrated seawater salt by electrodialysis [J]. Salt Science and Chemical Engineering, 2018 (3): 11-13.

[11] 高德顺. 我国北方地区海水淡化方式技术经济性比较研究 [D]. 大连: 大连理工大学(硕士学位论文), 2013 .

Gao D S. Comparative study on technical and economical characteristics of seawater desalination methods in northern China [D]. Dalian: Dalian University of Technology (Master's thesis), 2013.

[12] 张继立, 王益群. 我国海上风电区域开发方案浅析 [J]. 风能, 2018 (6): 62-68.

Zhang J L, Wang Y Q. Analysis on the development plan of offshore wind power region in China [J]. Wind Energy, 2018 (6): 62-68.

[13] 王朝乾, 田风超, 刘金荣. 浓海水电渗析制卤工艺研究 [J]. 盐业 与化工, 2015, 44(11): 39-43.

Wang C Q, Tian F C, Liu J R. Study on electrodialysis and halogenation process of concentrated seawater [J]. Journal of Salt and Chemical Industry, 2015, 44(11): 39-43.

[14] 姜学军. 东营协发公司年产50万吨离子膜烧碱项目财务分析 [D]. 青岛: 中国海洋大学(硕士学位论文), 2009.

Jiang X J. Financial analysis of the annual production of 500000 tons of ionic membrane caustic soda in Dongying association [D]. Qingdao: Ocean University of China (Master's thesis), 2009.

[15] 邓科, 张定明. 中国氯碱产业发展现状及未来竞争特点分析 [J]. 氯碱工业, 2013 (11): 5-19. 
Deng K, Zhang D M. Analysis of China's chlor-alkali industry development status and future competition characteristics [J]. Chlor-Alkali Industry, 2013 (11): 5-19.

[16] 赵学军, 袁玮. 氯碱行业技术及产品优化利用分析 [J]. 氯碱工 业, 2018 (9): 7-11.

Zhao X J, Yuan W. Analysis of chlor-alkali industry technology and product optimization and utilization [J]. Chlor-Alkali Industry, 2018 (9): 7-11.

[17] 刘国桢. 燃料电池技术及在氯碱工业的应用 [J]. 中国氯碱, 2016 (6): 1-5.

Liu G Z. Fuel cell technology and its application in chlor-alkali industry [J]. Chinese Chlor-Alkali, 2016 (6): 1-5. 